International Journal on Bioinformatics \& Biosciences (IJBB) Vol.3, No.3, September 2013

\title{
STRATEGY FOR ELECTROMYOGRAPHY BASED DIAGNOSIS OF NEUROMUSCULAR DISEASES FOR ASSISTIVE REHABILITATION
}

\author{
Sailesh Conjeti ${ }^{1}$ and Bijay Kumar Rout ${ }^{2}$ \\ ${ }^{1}$ Department of Electrical and Electronics Engineering, Birla Institute of Technology and \\ Science, Pilani, Rajasthan, India. \\ ${ }^{2}$ Department of Mechanical Engineering, Birla Institute of Technology and Science, \\ Pilani, Rajasthan, India.
}

\begin{abstract}
Assistive Rehabilitation aims at developing procedures and therapies which reinstate lost body functions for individuals with disabilities. Researchers have monitored electrophysiological activity of muscles using biofeedback obtained from Electromyogram signals collected at appropriate innervation points. In this paper, we present a comprehensive technique for detection of neuromuscular disease in a subject and a strategy for continuous therapeutic assessment using the Rehabilitation Assessment Matrix. The decision making tool has been trained using a wide spectrum of synthetic physiological data incorporating varying degrees of myopathy and neuropathy from beginning stages to acute. The statistical, spectral and cepstral features extracted from EMG have been used to train a Cascade Correlation Neural Network Classifier for disease assessment. The diagnostic yield of the classifier is $91.2 \%$ accuracy, $85.3 \%$ specificity and 91.35\% sensitivity. The strategy has also been extended to include isotonic contractions in addition to static isometric contractions. This comprehensive strategy is proposed to aid physicians plan and schedule treatment procedures to maximize the therapeutic value of the rehabilitation process.
\end{abstract}

\section{KEYWORDS}

Electromyography, Rehabilitation, Myopathy, Neuropathy and Cascade Correlation Neural Network.

\section{INTRODUCTION}

Assistive Rehabilitation of affected individuals aims at restoring original body functionality by compensating for the lost functions and thus providing opportunities to lead an independent life. Such procedures for neuromuscular rehabilitation often require design of manipulative physiotherapy procedures, following the detection of neuromuscular disease (NMD) in the subject. The biofeedback acquired from the patient is crucial to the design and execution of an effective medical rehabilitation scheme [1]. A complete and comprehensive assessment is often labour-intensive and expensive because the design and configuration of individualistic procedures require training of highly-skilled physiotherapists with appropriate expert knowledge. In this situation, Computer Aided Diagnostics of NMDs helps minimize observer bias, facilitates inter-subject comparison and aids the physicians to arrive at a more accurate diagnosis [2]-[3]. Neuromuscular facilitation mechanisms for individuals affected with NMDs must be designed considering individual's neurophysiology, motor-learning and motor development functions [4]. This work primarily focuses on two classes of NMD viz. Myopathy and Neuropathy. Myopathy refers to a medical condition where muscle weakness is observed due to reduced functionality and activation of muscle fibres for a particular nervous stimulation. Muscle cramps, stiffness 
International Journal on Bioinformatics \& Biosciences (IJBB) Vol.3, No.3, September 2013

and spasm are the usual reported symptoms associated with myopathic disorders [5]. Neuropathy, on the other hand, is a neurogenic condition resulting in loss of movement and haptic sensation owing to nervous damage. The reported symptoms include nerve pain, partial or complete paralysis, abnormal sensations and muscle weakness [6]. The treatment of neuromuscular diseases varies from medications, physical therapy, splinting and in acute cases even surgery is suggested. The treatment is often administered on the basis of the cause and origin of NMDs and the degree of its severity.

The presented work aims to develop an Electromyography based assessment approach for NMDs which is fast and reliable. The proposed methodologies would aid the physiotherapists in preparing an appropriate medical treatment scheme with proper scheduling of physiological exercise routines, thus maximizing the therapeutic value of the rehabilitation procedure instituted.
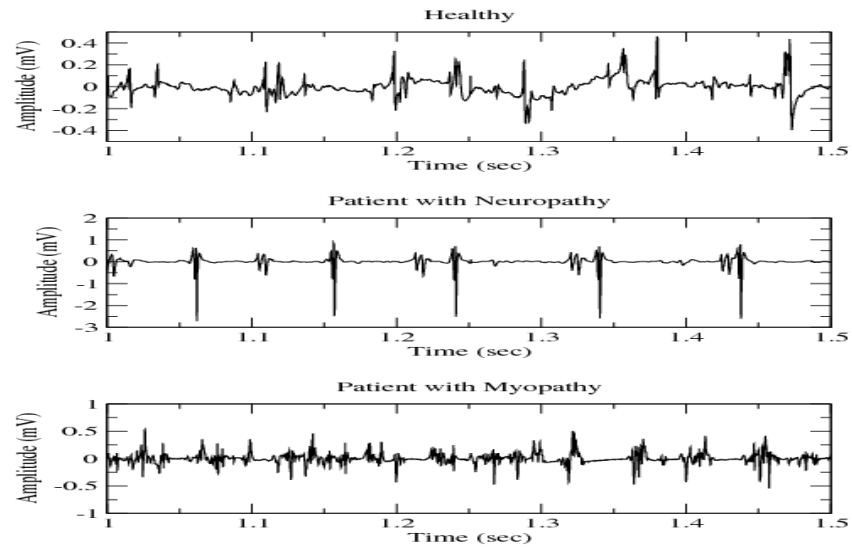

Figure 1. Intramuscular EMG Recordings (a) Normal Subject

(b) Neuropathic Subject and (c) Myopathic Subject

\section{ELECTROMYOGRAPHY AND APPLICATIONS IN NEUROMUSCULAR DISEASE DIAGNOSIS}

Researchers in the field of health monitoring have used the Electromyogram (EMG) signal for detection and monitoring of NMDs as they are accessible as bioelectric signals under direct volitional control [7]. EMG is a cumulative effect of the motor unit action potentials (MUAPs), generated by the motor neurons, which are responsible for actuating the skeletal muscles for support and motion of the human skeleton [8]. Electromyography signals acquired from appropriate muscular regions reflect on the muscle's tone, strength, abnormalities in reflexes, ideomotor and voluntary movements and postural equilibrium reactions [9]. Figure 1 illustrates a typical intramuscular EMG recorded from three subjects (a) Healthy, (b) Neuropathy and (c) Myopathy (Figure adapted from [10]).

Berzuini et al. investigated into the applicability of EMG signals collected from the right brachii muscles to detect neurogenic disorders. Variations observed in both time-domain and frequency domain parameters helped them to find topographical clusters in the multivariate space of EMG parameters corresponding to neuropathic subjects [11]. Pattiachis et al. investigated into applying Neural Network models of EMG diagnosis for detecting NMDs. They trained the networks using extracted morphological features of MUAP waveform after signal decomposition procedures on EMG [3]. These research works establish the suitability of EMG signals for detection of 
neuromuscular disease and development of a reliable rehabilitation strategy for a particular subject.

\section{REHABILITATION ASSESSMENT MATRIX}

The current styles of rehabilitation assessment forms used by medical practitioners in Physical Medicine and Rehabilitation (PMR) documents the patient's strengths, abilities, preferences, needs, findings, and recommendations for treatment. Inferences drawn from these ensure that appropriate rehabilitation procedures are administered on timely basis [12]. The Rehabilitation Assessment Matrix (RAM) has been developed to meet the need for comprehensive assessment of patient with NMDs which requires a quantitative progress chart to monitor the therapeutic value of the treatment being administered. The proposed design of such a matrix is shown in the Figure 2. The approach to obtain this RAM is discussed in the subsequent section. The integration of the proposed RAM to existing PMR assessment reports will provide the therapist measurable objectives for monitoring the patient's progress during the course of assistive rehabilitation.

A motor unit refers to an $\alpha$-motor neuron of the Central Nervous System and the set of muscle fibres it innervates. When a motor unit (MU) is recruited, it contributes a quantum of force to muscular contraction [13]. The changes in the active MU density are attributable to age, buildup, disease and injury. It is observed that though MU density reduces with age, it is not as severe as the effects due to NMDs. It is observed to be a very useful parameter to monitor neurogenic disease progression, motor-neuron death rate and motor development improvements during rehabilitation [14]. The active MU density in Biceps Brachii muscle has been reported as average of 109 per $\mathrm{mm}^{2}$ (Std. Dev.: 53 per $\mathrm{mm}^{2}$ ) for a stroke related myopathy patient and about 153 per $\mathrm{mm}^{2}$ (Std. Dev.: 38 per $\mathrm{mm}^{2}$ ) for a normal subject [15]. The number of active motor units per unit area, inferred from the EMG has been recorded in the X-axis of the RAM. The axis has been sub-divided into 8 classes with a class width of 15 active MUs per $\mathrm{mm}^{2}$ (shown in Fig. 2). However, it must be noted that this work does not delve into Motor Unit Number Estimation (MUNE) methods. Researchers have developed enhanced statistical approaches for MUNE like Bayesian Estimators [16], Poisson Techniques [17], Higher Order Statistics [18] and SpatioTemporal Summation approaches [19].

\begin{tabular}{|c|c|c|c|c|c|c|c|c|c|}
\hline \multicolumn{10}{|c|}{ Rehablitation Assessment Matrix } \\
\hline \multirow{2}{*}{\multicolumn{3}{|c|}{$\stackrel{\text { No. of Active Motor Units }}{\longrightarrow}$}} & & & & & & & \\
\hline & & & $<90$ & $90-105$ & $105-120$ & $120-135$ & $135-150$ & $150-165$ & $>180$ \\
\hline & \multicolumn{2}{|c|}{ Loss Fraction } & & & & & & & \\
\hline \multirow[t]{2}{*}{ Extremely Myopathic } & $1.0 \mathrm{M}$ & \multirow{3}{*}{$\stackrel{a}{i}$} & & & & & & & \\
\hline & $0.8 \mathrm{M}$ & & & ..... & ............ & \multicolumn{3}{|c|}{ Myopathy Progress Curve } & \\
\hline \multirow[t]{2}{*}{ Moderately Myopathic } & $0.6 \mathrm{M}$ & & & & & & $/$ & & \\
\hline & $0.4 \mathrm{M}$ & \multirow[t]{2}{*}{+} & & & & & & & \\
\hline Mildly Myopathic & $0.2 \mathrm{M}$ & & & & & & $\cdots$ & & \\
\hline Normal Body Functioning & 0.0 & \multirow{6}{*}{ : } & & & .................. & …............ & $\ldots \ldots \ldots$ & 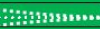 & $\rightarrow$ \\
\hline \multirow[t]{2}{*}{ Mildly Neuropathic } & $0.2 \mathrm{~N}$ & & & & & & & & \\
\hline & $0.4 \mathrm{~N}$ & & & & & & & & \\
\hline \multirow[t]{2}{*}{ Moderately Neuropathic } & $0.6 \mathrm{~N}$ & & & & & & & & \\
\hline & $0.8 \mathrm{~N}$ & & & .... & ......... & \multicolumn{3}{|c|}{ Neuropathy Progress Curve } & \\
\hline Extremely Neuropathic & $1.0 \mathrm{~N}$ & & & & & & & & \\
\hline \multicolumn{2}{|c|}{$\begin{array}{c}\text { Legend } \\
\text { Degree of Neuromuscular Disease }\end{array}$} & & \multicolumn{7}{|c|}{$\begin{array}{l}\text { Low : Healthy Individual with Normal Functioning } \\
\text { Moderate : Correctable through proper rehabilitation exercises } \\
\text { High : Requires Support of Orthosis } \\
\text { Acute :Complicated Stage } \\
\end{array}$} \\
\hline
\end{tabular}

Figure 2. Rehabilitation Assessment Matrix

The $\mathrm{Y}^{+}$-axis of the RAM (shown in Figure 2) refers to the myopathic affected fibre fraction representing the degree of myopathy whereas the $\mathrm{Y}^{--}$-axis refers to the neuropathic motor unit 
International Journal on Bioinformatics \& Biosciences (IJBB) Vol.3, No.3, September 2013

loss fraction corresponding to the degree of neuropathy. As treatment progresses, the subject's improvement line, both Myopathy Progress Curve and Neuropathy Progress Curve (Sample highlighted in Figure 2) moves towards the Normal body functioning line. The pattern followed in the RAM reflects the subject's motor learning ability and can provide useful insights into the nature of exercise the physiotherapist can administer to the subject. If the subject's curve is not progressive as desired, the physiotherapist's may be alerted to a need for change in the exercise routine and treatment being administered.

\section{Physiological data acQuisition, Pre-Processing and Feature EXTRACTION}

The Electromyogram signals were synthetically generated using a physiological model developed by Wright and Stashuk. This model generates EMG signals consistent with those acquired through intramuscular needle electrodes from the limb muscles (including biceps brachii) of human subjects [20]. Though the Kinesiological EMG acquired through intramuscular electrodes is invasive over surface electrodes, it is preferred for neuromuscular disease prognosis due to its increased signal specificity and more selective recording to characterize the muscle of interest [21]. The EMG signal was generated at $31,250 \mathrm{~Hz}$ and band pass filtered from 10 to $10,000 \mathrm{~Hz}$ to remove enhance signal characteristics. The selected muscle and motorneuron pool settings of the Wright-Stashuk model correspond to the biceps brachii muscle of a human subject, simulating a constant force isometric contraction without fatigue or spasm. Isometric contractions are static muscle contractions which happen without any appreciable decrease in fibre length and change in the distance between point of electrode insertion and origin of EMG signals. The subject-age and gender were not fixed during each simulation to minimize any fixed-variable bias and enhance data set universality. The simulated intra-muscular needle electrode configuration, contraction type and the muscle model parameters are described in Table 1.

Table 1. Description of parameters for EMG Generation

\begin{tabular}{|c|c|c|}
\hline S.N. & $\begin{array}{l}\text { Type of } \\
\text { Parameter }\end{array}$ & Description \\
\hline 1 & $\begin{array}{l}\text { Electrode } \\
\text { Configuration }\end{array}$ & $\begin{array}{l}\text { Differential Electrode Configuration } \\
\text { Detection Surfaces Dimension: Length: } 1.0 \mathrm{~cm} \text {; Width: } \\
1.2 \mathrm{~mm} \text {; Separation: } 1.0 \mathrm{~cm} \\
\text { Bandwidth: } 20-500 \mathrm{~Hz} \text { with a } 40 \mathrm{~dB} / \mathrm{decade} \text { roll-off. } \\
\text { Common Mode Rejection Ratio: }>80 \mathrm{~dB} \text { Noise: }<2 \mu \mathrm{V} \text { rms } \\
(20-400 \mathrm{~Hz}) \text { Input Impedance: }>100 \mathrm{M} \Omega\end{array}$ \\
\hline 2 & Electrode Location & $\begin{array}{l}\text { Middle line of Muscle Belly between the myotendonous } \\
\text { junction and nearest innervation zone. }\end{array}$ \\
\hline 3 & $\begin{array}{l}\text { Muscle Model } \\
\text { Parameters }\end{array}$ & $\begin{array}{l}\text { Contraction Level ( \% MVC): 5-50\% } \\
\text { No. of Active MU Density: } 75-180 \text { per } \mathrm{mm}^{2}\end{array}$ \\
\hline 4 & $\begin{array}{l}\text { Disease } \\
\text { Parameters }\end{array}$ & $\begin{array}{l}\text { Neuropathic Motor Unit Loss Fraction: } 0.0 \text { (Healthy)-1.0 } \\
\text { (Extreme) Step Size: } 0.25 \\
\text { Myopathic Fiber Affected Fraction:0.0(Healthy)-1.0 } \\
\text { (Extreme) Step Size: } 0.25\end{array}$ \\
\hline
\end{tabular}

\subsection{Synthetic EMG Generation from Wright-Stashuk Model}

A total of 2000 sample-waveforms (10 Contraction-levels $\times 8$ Classes of Active MU Density $\times 5$ Classes of Myopathy $\times 5$ Classes of Neuropathy) were synthetically generated for 5 seconds at a sample rate of $31,250 \mathrm{~Hz}$. The objective behind introducing high degree of inter-simulation variability is to develop a universal approach that can be readily extended to medical domain. 
International Journal on Bioinformatics \& Biosciences (IJBB) Vol.3, No.3, September 2013

The algorithms and codes for analysis have been written in MATLAB R2010b® and use the inbuilt Statistics, System Identification and Neural Network Toolboxes.

\subsection{Physiological Feature Extraction}

In the present case, the generated signals were filtered for suppressing signal aliasing and motion artifacts using a $5^{\text {th }}$ order Savitsky Golay Filter. The filter fits a $5^{\text {th }}$ order polynomial in a window of 41 data points. It reduces noise while preserving the waveform's shape, structure, the relative maxima, minima and width [22]. The smoothened signal representing muscle force for the three real-life test signals has been shown in the Figure 3.The filtered muscle data is partitioned into static windows and the features extracted from each window will constitute the characteristic feature vector. The optimal window size for this particular application is expected to vary between $100 \mathrm{~ms}$ and $1 \mathrm{~s}$. Therefore, classifier's decision making performance would be evaluated for good trade-off between accuracy and specificity, over 10 windows from $100 \mathrm{~ms}$ to $1 \mathrm{~s}$ in steps of $100 \mathrm{~ms}$. The extracted features are explained in the following subsections.
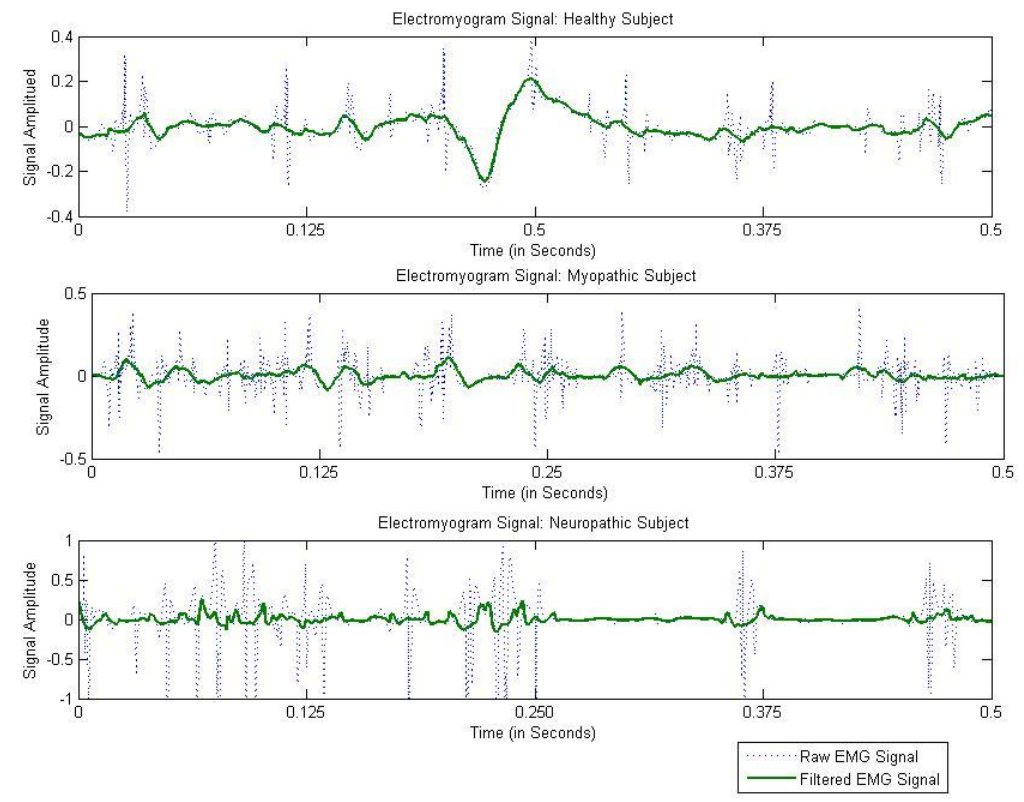

Figure 3. Savitsky-Golay Filtering

\subsubsection{Statistical Features}

These features help in assessment of uncertainty associated with physiological signal. De Luca et al. demonstrated that time-domain statistical features of EMG are influenced by MU firing rate, number of detected active MU, the MU activation potential, duration, waveform morphology and the recruitment stability [9]-[23]. The statistical features extracted include: Global Maxima, Global Minima, Mean, Standard Deviation, Energy, Time Duration, Bandwidth, Time Bandwidth Product, $3^{\text {rd }}$ order moment, $4^{\text {th }}$ order moment, $5^{\text {th }}$ order moment, Root Mean Square Value, Kurtosis and Skewness as tabulated in Table 2.

Table 2. Statistical Features of EMG Signal

\begin{tabular}{|c|l|l|c|l|l|}
\hline S.N. & Feature Name & Formula & S.N. & Feature Name & Formula \\
\hline 1 & $\begin{array}{l}\text { Global } \\
\text { Maxima }\end{array}$ & $\operatorname{Max}(\forall x[n])$ & 8 & $\begin{array}{l}\text { Time } \\
\text { Bandwidth Product }\end{array}$ & $T D \times B W$ \\
\hline
\end{tabular}


International Journal on Bioinformatics \& Biosciences (IJBB) Vol.3, No.3, September 2013

\begin{tabular}{|c|c|c|c|c|c|}
\hline 2 & Global Minima & $\operatorname{Min}(\forall x[n])$ & 9 & $\begin{array}{l}3^{\text {rd }} \text { Order } \\
\text { Moment }\end{array}$ & $\frac{1}{N} \sum_{i=1}^{N} x[i]^{3}$ \\
\hline 3 & Mean & $\vec{x}=\frac{1}{N} \sum_{i=1}^{N} x[i]$ & 10 & $\begin{array}{l}4^{\text {th }} \text { Order } \\
\text { Moment }\end{array}$ & $\frac{1}{N} \sum_{i=1}^{N} x[i]^{4}$ \\
\hline 4 & $\begin{array}{l}\text { Std. } \\
\text { Dev. }\end{array}$ & $s=\sqrt{\frac{1}{N} \sum_{i=1}^{N}(x[i]-\bar{x})^{2}}$ & 11 & $\begin{array}{l}5^{\text {th }} \text { Order } \\
\text { Moment }\end{array}$ & $\frac{1}{N} \sum_{i=1}^{N} x_{[i]^{5}}$ \\
\hline 5 & Energy & $\sum_{i=1}^{N} x[i]^{2}$ & 12 & $\begin{array}{l}\text { Root } \\
\text { Mean Square }\end{array}$ & $\sqrt{\frac{1}{N} \sum_{i=1}^{N} x_{[i]^{2}}^{2}}$ \\
\hline 6 & Time Duration & $T D=\left(\frac{\sum_{i=1}^{N}(i-\vec{i})^{2} x[i]^{2}}{\sum_{i=1}^{N} x[i]^{2}}\right)^{1 / 2}$ & 13 & Kurtosis & $\frac{\sum_{i=1}^{N}(x[i]-\vec{x})^{4}}{(N-1) s^{4}}$ \\
\hline 7 & Bandwidth & $B W=\frac{1}{2 \pi}\left(\frac{\left.\sum_{i=2}^{N}(x i]-x[i-1]\right)^{2}}{\left.\sum_{i=1}^{N} \sum^{2}\right)^{1 / 2}}\right)^{N}$ & 14 & Skewness & $\frac{\sum_{i=1}^{N}(x[i]-\vec{x})^{3}}{(N-1) s^{3}}$ \\
\hline
\end{tabular}

\subsubsection{Spectral Features}

In studies on rehabilitation, it is desirable to predict fatigue before it commences so that appropriate remedies may be adopted. Researchers have used the contractile force approach for evaluating fatigue points when the subject undergoes sustained contraction. Changes in the muscle force and the monitored torque indicate progress of fatigue with time. But, this method is considered inefficient as force and torque provide only a general overview of the entire muscle and not the individual motor units. In this context, the spectral modification during compression, along with the alteration of the skewness of the EMG waveform is acceptable as a Fatigue Index. EMG gives a more holistic view on muscle fatigue as individual skeletal muscle can be monitored continuously from the point of onset of the contraction. The Figure 4 below illustrates the factors of EMG which influence the spectral modification property and its interrelationships.

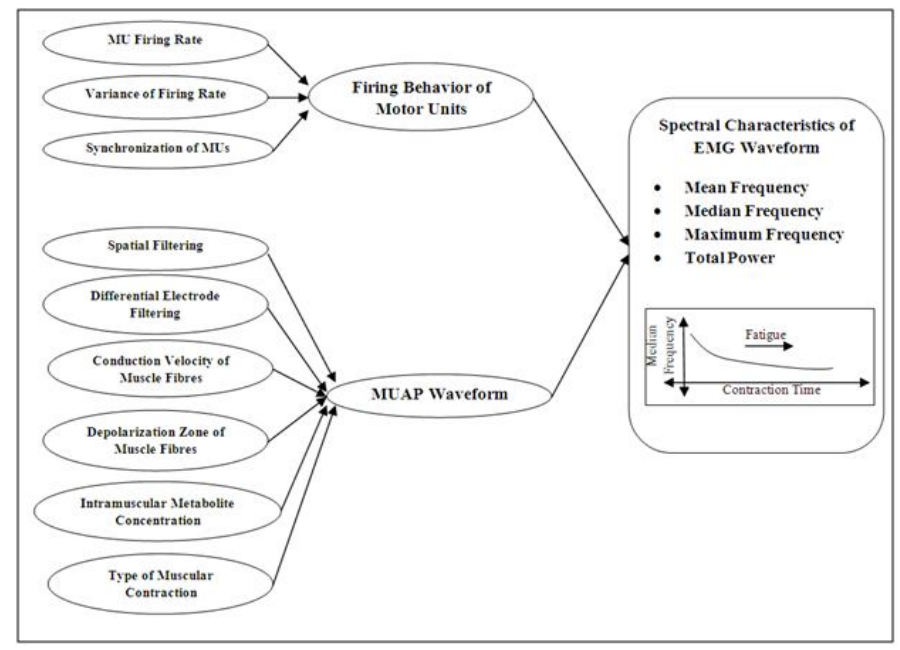

Figure 4. Factors influencing spectral characteristics of EMG waveform

Researchers have observed spectral modifications in the power spectrum of EMG acquired during tetanic muscular contractions (Tetanic refers to sustained muscle contraction without rest intervals). The skewness of the MUAP waveform is observed to alter with increasing fatigue and changes in muscle biochemistry due to continuous accumulation of lactic acid in the muscle cell (Anaerobic Respiration) [24]. The power spectrum of EMG signals was estimated using the Lomb periodogram approach because of its robustness to motion artifacts and missed data points 
and lesser computational complexity for real-life biomedical applications [25]. Let $S(f)$ represent the Lomb periodogram of the EMG signal over an input range of $f: 10 \mathrm{~Hz}$ to $10000 \mathrm{~Hz}$ (Frequency Resolution $\Delta f: 1 \mathrm{~Hz}$ ). The spectral features extracted are the mean, median and the maximum frequency which are given by formulae (1)-(3) respectively.

$$
\begin{aligned}
f_{\text {mean }} & =\frac{\sum_{f=10}^{10000} f \times s(f) \times \Delta f}{\sum_{f=10}^{10000} s(f) \times \Delta f} \\
f_{\text {median }} & =\frac{1}{2} \sum_{f=10}^{10000} s(f) \times \Delta f \\
f_{\text {max imum }} & =\arg \forall_{f} \max (s(f))
\end{aligned}
$$

\subsubsection{Cepstral Features}

Cepstral Coefficients are calculated from the inverse Fourier Transform of the logarithmic power spectrum of EMG. Yoshikawa et al. established that the lower order Cepstral coefficients extracted from EMG can be used in robust classification of hand motions [26].These cepstral coefficients are derived from the $10^{\text {th }}$ order autoregressive model of the filtered EMG signal. Let $x(k)$ represent the filtered EMG signal, $a_{i}$ is the $i^{\text {th }}$ coefficient of the $M$-order autoregressive model and $e(k)$ is the white noise in the system (refer Formula (4)). The cepstral coefficients $c_{i}$ $(i=1: 5)$ are derived from $a_{i}$ using recursive formulae (5)-(6).

$$
\begin{aligned}
& x(k)=\sum_{i=1}^{M=10} a_{i} x(k-i)+e(k) \\
& c_{1}=-a_{1} c_{n=1}^{i-1}\left(1-\frac{n}{i}\right) a_{n} c_{i-1} \quad \text { where } 1<i \leq M(=5) \\
& c_{i}=-a_{i}-\sum_{n=1}
\end{aligned}
$$

\section{NEUROMUSCULAR DISEASE DETECTION USING CASCADE CORRELATION NEURAL NETWORK}

From a single window of generated Synthetic EMG signals, 22 features (14-Statistical + 3Frequency +5 -Cepstral) are extracted to form the representative feature vector. For training the classifier, to detect NMD, the concatenated feature vectors extracted divided in the ratio of 60:20:20 for training, cross-validation and testing respectively. The extracted EMG feature vector was divided into two classes based on degree of myopathy and neuropathy viz. Healthy and Unhealthy i.e. with NMD. The Healthy data comprises of signal samples with Myopathic Fiber Affected Fraction (MFAF) : 0.3 and Neuropathic Motor Unit Loss Fraction (NMULF) : $<0.3$. The Unhealthy data comprises of signal samples of myopathic nature with MFAF: $\geq 0.3$ and neuropathic samples with NMULF: $\geq 0.3$. These values were decided as heuristic estimates on the basis of review by Visser et al. on critical illness polyneuropathy and myopathy [27].

Traditional Back-propagation algorithm for Neural Network training is slow in training higherorder networks due to the step-size and moving target problems. Cascade Correlation Neural (CCN) Network exhibit a learning time of the order of $N \log N$ and is comparatively over 10 times faster than the back-propagation algorithm. The dynamic creation of hidden units, broadcast of residual error to all candidates without back-propagation and use of incremental learning ensures the CCN's applicability to higher order classification problems [28]. Owing to the extensive variability incorporated in the training data, there exists a potential problem of classifier convergence into local optima. Therefore, a faster convergence training algorithm which ensures global optima must be preferred in this application and hence CCN network is chosen over 
International Journal on Bioinformatics \& Biosciences (IJBB) Vol.3, No.3, September 2013

traditional back-propagation networks. The $\mathrm{CCN}$ network employed the training algorithm developed by [29] using the MATLAB Neural Network Toolbox.CCN Network utilizes cascade neural network architecture and learning algorithm where the hidden candidate units are added sequentially with the objective of finding the best number of hidden layers and neurons with reliable learning and generalization capabilities i.e. optimal bias-variance trade-off [30]. The connections from the input layer to the hidden layer are frozen whereas the cascade connections and the connections between hidden and output layers are trainable. The network-topology chosen is a cascade-feed-forward network with one hidden layer and cascade connections existing from the input to the output layer. The tan-sigmoid transfer function is employed at the hidden layer and at the output layer a linear transfer function is used.

Table 3. Cascade Correlation Neural Network Architecture

\begin{tabular}{|c|c|c|}
\hline S.N. & Parameter & Characteristic Adopted \\
\hline a. & No of Layers & - 2 Layer Network with 1 Hidden \\
\hline b. & Hidden Neurons & - 10 in Layer 1 and 3 in Output \\
\hline c. & Training Function & $\begin{array}{l}\text { - Levenberg-Marquardt algorithm } \\
\text { - } \sigma=5.0 \mathrm{E}-05 \\
\text { - } \lambda=5.0 \mathrm{E}-07\end{array}$ \\
\hline d. & Learning Function & $\begin{array}{l}\text { - Gradient Ascent Momentum } \\
\text { - Learning Rate= } 0.01 \\
\text { - } \text { Momentum Constant= } 0.9\end{array}$ \\
\hline e. & Weight Initialization & - Nyugen-Widrow Method \\
\hline f. & Transfer Function & $\begin{array}{l}\text { - Tan Sigmoid at Layer } 1 \\
\text { - Linear at Output Layer } \\
\end{array}$ \\
\hline g. & Termination & $\begin{array}{l}\text { - Maximum No. of Epochs: } 1000 \\
\text { - Max. Validation Failures: } 15 \\
\text { - Max. Time to Train: } 300 \text { seconds }\end{array}$ \\
\hline
\end{tabular}

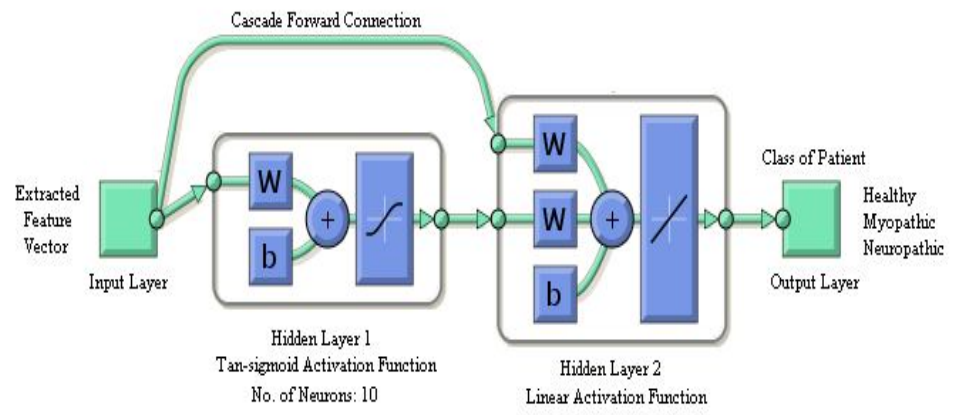

Figure 5. Network Architecture of Cascade Correlation Neural Network 
International Journal on Bioinformatics \& Biosciences (IJBB) Vol.3, No.3, September 2013

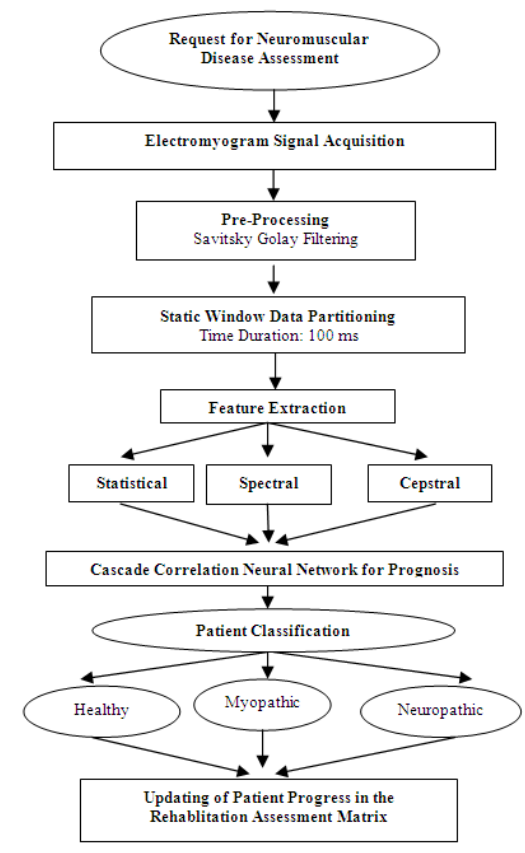

Figure 6. Flowchart for Evaluation of Neuromuscular Pathology using proposed strategy.

\section{INVESTIGATIONS INTO APPLICABILITY OF ISOTONIC CONTRACTIONS FOR NEUROMUSCULAR DISEASE DIAGNOSIS}

In the present study, it is envisaged that for comprehensive diagnosis of NMDs, Range of Motion (ROM) studies and dynamic changes during the course of rehabilitation exercise must also be integrated. Including dynamic changes noticeable during isotonic contractions like muscle flexion, extension, pronation etc in NMD diagnosis would enable a complete assessment of motor ability and neuromuscular coordination in the muscles. This section aims to investigate the applicability of isotonic contractions to NMD diagnosis and hence develop an experimental protocol for the same.

For the present study, the subject considered is a 20 year old healthy, average-built man with no history of neuromuscular diseases. It is assumed that such a subject would give a representative understanding of human muscle activation pattern during rehabilitation exercises. The EMG signals were acquired from the Biceps Brachii muscles using the Myotrace 400 Acquisition setup from Noraxon at a sample rate of $20 \mathrm{KHz}$ [31]. The experiment consisted of three trials of elbow flexion and extension cycles with variations in cycle speed. Trial 01 is normal speed full range of motion

(ROM: $20^{\circ}-140^{\circ}$ of elbow angle in sagittal plane) exercise with Average time for Flexion (ATF): 1.4s, Average time for Extension (ATE): $1.6 \mathrm{~s}$ and Total Cycles (TC): 20 cycles. Trial 02 is slow speed full ROM exercise with ATF: $2.8 \mathrm{~s}$, ATE: $3.0 \mathrm{~s}$ and TC: 13 cycles. Trial 03 is slow speed ROM exercise with ATF: $0.5 \mathrm{~s}$ ATE: $0.8 \mathrm{~s}$ and TC: 11 cycles. It must be noted that each trial was followed by 5 minutes of rest period to remove any effects of muscle fatigue or stress acquired due to the exercises. The data collected under these trials would be tested against the CCN Classifier for NMD diagnosis developed in Section 5 and its performance would be presented in Section 7. Figure 6 illustrates the complete flow diagram of the proposed strategy as established in Sections 4-6. 
International Journal on Bioinformatics \& Biosciences (IJBB) Vol.3, No.3, September 2013

\section{RESULTS AND DISCUSSION}

To analyze the network performance over different chosen window sizes, performance metrics like the training accuracy $A c c_{T r}$, training specificity $S p_{T r}$, the testing accuracy $A c c_{T s}$ and the testing specificity $S p_{T s}$ are extracted from the individual training and testing data confusion matrices generated after $\mathrm{CCN}$ training. For the purposes of optimizing the window size, we have to define a desirability function $D\left(x_{i}\right)$ for the particular application. This function is defined in [32] as the min-max normalized value (refer Formula (7)) of the observed performance parameter and the geometric mean of desirabilities is to be employed to optimize a multiparameter problem.

$$
D\left(x_{i}\right)=\frac{\text { Observed }\left(x_{i}\right)-\text { Minimum }(\forall x)}{\text { Maximum }(\forall x)-\text { Minimum }(\forall x)}
$$

In this particular application, we define $D\left(A c c_{T r}\right), D\left(S p_{T r}\right), D\left(A c c_{T s}\right)$ and $D\left(S p_{T s}\right)$ as per Formula (8) and the cumulative desirability function $D_{\text {Cum }}$ as

$$
D_{C u m}=\sqrt[4]{D\left(A c c_{T r}\right) \times D\left(S p_{T r}\right) \times D\left(A c c_{T s}\right) \times D\left(S p_{T s}\right)}
$$

Table 4. Cascade Correlation Neural Network Classifier Performance for various window sizes.

\begin{tabular}{|l|l|l|l|l|l|}
\hline S. & Window Size & Training Data & Testing Data & \\
\cline { 2 - 6 } $\mathbf{N}$. & & Accuracy Acc $_{\text {Tr }}$ & Specificity $_{\text {Sp }}^{\text {Tr }}$ & Accuracy Acc $_{\text {Ts }}$ & ${\text { Specificity } \mathbf{S p}_{\text {Ts }}}$ \\
\hline 1 & $100 \mathrm{~ms}$ & 0.965 & 0.815 & 0.912 & 0.742 \\
\hline 2 & $200 \mathrm{~ms}$ & 0.953 & 0.824 & 0.907 & 0.751 \\
\hline 3 & $300 \mathrm{~ms}$ & 0.942 & 0.846 & 0.914 & 0.772 \\
\hline 4 & $400 \mathrm{~ms}$ & 0.912 & 0.853 & 0.897 & 0.785 \\
\hline 5 & $500 \mathrm{~ms}$ & 0.886 & 0.881 & 0.864 & 0.797 \\
\hline 6 & $600 \mathrm{~ms}$ & 0.874 & 0.894 & 0.854 & 0.813 \\
\hline 7 & $700 \mathrm{~ms}$ & 0.885 & 0.912 & 0.831 & 0.821 \\
\hline 8 & $800 \mathrm{~ms}$ & 0.861 & 0.934 & 0.842 & 0.834 \\
\hline 9 & $900 \mathrm{~ms}$ & 0.854 & 0.941 & 0.825 & 0.854 \\
\hline 10 & $1 \mathrm{~s}$ & 0.832 & 0.948 & 0.817 & 0.864 \\
\hline
\end{tabular}

From Table 4, the desirability of individual performance metrics is calculated using Formula (7) and the cumulative desirability using Formula (8). Figure 7 shows the plots of individual desirabilities $D\left(A c c_{T r}\right), D\left(S p_{T r}\right), D\left(A c c_{T s}\right)$ and $D\left(S p_{T S}\right)$ and cumulative desirability $D_{C u m}$ for each of the window-size considered. The approach resulted in an optimal window size of $400 \mathrm{~ms}$ with a maximum desirability of 0.4278 (shown in Figure 7). For this optimal window size of 12500 samples at a rate of $31.25 \mathrm{KHz}$, the $A c c_{T r}$ measured was $91.2 \%, S p_{T r}$ measured was $85.3 \%, A c c_{T s}$ is observed as $89.7 \%$ and $S p_{T s}$ was measured to be $78.5 \%$. These represent the performance parameters of an optimal CCN network for the optimal window size chosen for this application. 
International Journal on Bioinformatics \& Biosciences (IJBB) Vol.3, No.3, September 2013

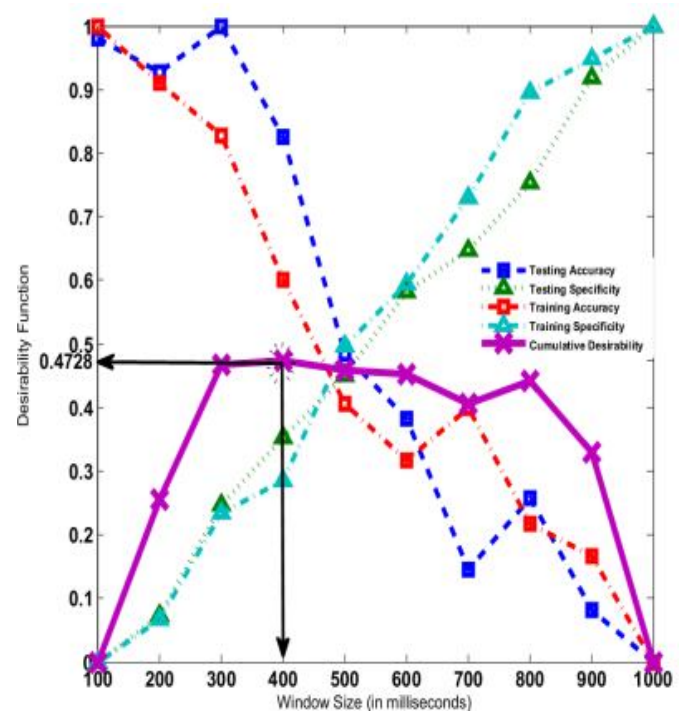

Figure 7. Functions $D\left(A c c_{T r}\right), D\left(S p_{T r}\right), D\left(A c c_{T s}\right)$ and $\left(S p_{T s}\right)$ and $D_{c u m}$ for different window sizes in Cascade Correlation Neural Network

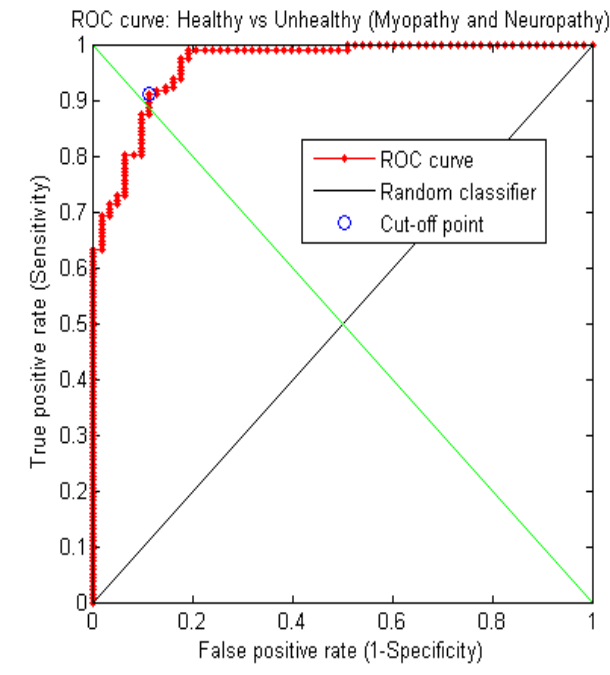

Figure 8. Receiver Operating Characteristics of Optimal CCN Classifier

Receiver Operating Characteristics (ROC) curves has been used to evaluate the trade-off between sensitivity and specificity in Classifier design. The Area under the Curve (AUC) statistic, used for model comparison measures the specificity-sensitivity tradeoff. The ROC curve obtained for the optimum CCN Classifier for the most desirable window size of $400 \mathrm{~ms}$ is shown in Figure 8. It was observed that the AUC was 0.9552 for a tolerance of \pm 0.05 . As the AUCStatistic value is $\geq 0.9$ the classification method is considered an 'Excellent' test [33]. The cut-off point for best sensitivity and specificity corresponded to a value of 0.452 . This implies if the network's output value is $\leq 0.452$ the subject is classified as Healthy and $>0.452$ the subject is classified as Unhealthy. For such a cutoff, the sensitivity of classifier performance was observed as $91.35 \%$ and the overall specificity was $85.3 \%$. 
International Journal on Bioinformatics \& Biosciences (IJBB) Vol.3, No.3, September 2013

Further, the designed classifier for the optimal window size of $400 \mathrm{~ms}$ is tested against the human Kinesiological EMG data acquired from Physionet signal database and the observed outputs from the network are tabulated in Table 5. The data includes intracellular EMG signals acquired from the biceps brachii muscle from three human subjects: a 44-year old man without any medical history of neuromuscular disease, a 62-year old man with chronic lower back pain and neuropathy and a 57-year old man with myopathy [10]. As observed from ROC analysis, the network's output cut-off of 0.452 is used to demarcate the healthy subject from an unhealthy subject. It is hence observed that the developed classifier accurately classifies the presented human subject acquired signals into their respective classes thus provides a proof-of-concept for the presented approach.

Table 5. Results of Testing Real-Life EMG Data Against the Trained CCNN

\begin{tabular}{|l|l|l|l|l}
\hline S.N. & Class of Patient & Target Data & Avg. Output & Network Output \\
\hline 1. & H & 0.0 & 0.1186 & H \\
\hline 2. & UH: M & 1.0 & 0.7874 & UH \\
\hline 3. & UN: N & 1.0 & 0.8755 & UH \\
\hline \multicolumn{2}{|c|}{ Legend: H: Healthy UH: Unhealthy M: Myopathic N: Neuropathic } \\
\hline
\end{tabular}

For investigating the extendibility of CCNN Classifier technique to perform NMD diagnosis using dynamic isotonic contractions, the 22-attribute feature vector was extracted for a time window of $400 \mathrm{~ms}$ from the data acquired using protocols described in Section 6. Since the subject is a healthy subject, the performance analysis metrics for testing the classifier here were decided as the output mean square error (MSE) as against accuracy and specificity. The observations are tabulated in Table 6. The data from Trail 01 and Trial 02 were diagnosed correctly and the misclassification of Healthy into Unhealthy for Trial 03 must be noted. Since the performance of Trial 02 data is better that Trail 01 and Trial 03 , it is proposed that for extending the CCNN Classifier to dynamic isotonic contractions, the EMG data must be acquired through slow and steady flexion and extension motions.

Table 6. Results of Testing CCNN Against Isotonic Contractions

\begin{tabular}{|l|l|l|l|l|l|l|}
\hline S.N. & Trial & Speed & Target Data & Avg. Output & Diagnosis & MSE Observed \\
\hline 1. & T 01 & Normal & 1.0 & 0.7421 & Healthy & $3.211 \mathrm{E}-01$ \\
\hline 2. & T 02 & Slow & 1.0 & 0.8745 & Healthy & $1.602 \mathrm{E}-01$ \\
\hline 3. & T 03 & Fast & 1.0 & 0.4352 & Unhealthy & $6.458 \mathrm{E}-01$ \\
\hline
\end{tabular}

In Table 7, similar works for NMD detection available in literature are presented. Although direct comparison is not feasible, the proposed strategy compares well since it is trained using EMG data incorporating varied levels of disease severity both in neuropathy and myopathy and the learning technique for neural network training (Cascade Correlation) ensures design of an optimal classifier for the application.

Table 7. Performance Evaluation of Present Technique vs. Existing Literature

\begin{tabular}{|l|l|l|l|}
\hline S.N. & Author/Research Group & Technique & Classification Accuracy \\
\hline 1. & Chistoudoulou et al. [34] & Modular Neural Network & $79.6 \%$ \\
\hline 2. & Pattichis et al. [3] & $\begin{array}{l}\text { Feed-forward Network+ } \\
\text { Self Organizing Maps }\end{array}$ & $80 \%$ \\
\hline 3. & H.B. Xie et al.[35] & Support Vector Machine & $82.4 \%$ \\
\hline 4. & H.B. Xie et al.[36] & Hybrid Neuro-Fuzzy Systems & $88.58 \%$ \\
\hline 5. & This Work & Cascade Correlation Neural Network & $\begin{array}{l}91.2 \% \text { (Training) } \\
89.7 \% \text { (Testing) }\end{array}$ \\
\hline
\end{tabular}


International Journal on Bioinformatics \& Biosciences (IJBB) Vol.3, No.3, September 2013

\section{CONCLUSIONS AND FUTURE WORK}

The proposed diagnostic system for NMD detection utilizes the Cascade Correlation Neural Network learning methodology. The optimal window size for diagnosis was inferred as $400 \mathrm{~ms}$ and the classification using CCN Networks resulted in 91.2\% accuracy, $85.3 \%$ specificity and $91.35 \%$ sensitivity for training data. For testing data, the diagnostic yield was $89.7 \%$ accuracy and an acceptable specificity of $78.5 \%$. The proof-of-concept for extending the CCN classifier to real-life isometric contraction is established by testing on real-life kinesiological data. Investigations on data acquired using isotonic elbow flexion and extension contractions established that this method can be extended to dynamic studies. Further, integration of the proposed Rehabilitation Assessment Matrix with existing Physical Medicine and Rehabilitation practices will provide measurable objectives for therapists to monitor the patient's progress and help in preparing an appropriate medical treatment scheme to maximize the therapeutic value of the rehabilitation process.

In the future, decision support systems to for NMD diagnosis will be developed which incorporate a multimodal diagnosis approach fusing EMG data with inferences from biochemical analysis, neuropathology and clinical observations. This work incorporates the need for using data from subjects with different stages of NMDs and is envisaged as a step forward towards realizing a holistic and reliable EMG based NMD diagnostic system which can aid the physician in his decision making process.

\section{REFERENCES}

[1] S. Komada, Y.Hashimoto, N. Okuyama, T. Hisada, and J. Hirai, "Development of a Biofeedback Therapeutic-Exercise-Supporting Manipulator," IEEE Trans. on Industrial Electronics, vol. 56, no. 10, pp. 3914-3920, Oct. 2009.

[2] S.B. O'Sullivan, and T.J. Schmitz, "Physical Rehabilitation: Assessment and Treatment" , 2nd ed., F.A. Davis Company, Philadelphia, PA, 1988.

[3] C.S. Pattichis, C.N. Schizas, and L.T. Middleton, "Neural Network Models in EMG diagnosis," IEEE Trans. in Biomedical Engg., vol. 42, no. 5, pp.486-496, May 1995.

[4] T. Hisada,N. Okuyama, S. Komada, and J. Hirai, "Preliminary study on robotic exercise therapy," Proc. 30th Annual Conf. IEEE Industrial Electronics Society, vol. 3, pp. 2780-2785, Nov. 2004.

[5] J. Chawla, "Stepwise Approach to Myopathy in Systemic Disease," Frontiers in Neurology, vol. 2(49), August 2011.

[6] M.E. Shy, "Peripheral neuropathies," Cecil Medicine, Chapter 446, 23rd ed. Philadelphia, Saunders Elsevier, 2007.

[7] Stanford V. "Biosignals offer potential for direct interfaces and health monitoring," Pervasive Computing, vol. 04, pp. 99-103, 2004.

[8] J.V. Basmajian and C. J. de Luca, "Muscles Alive - The Functions Revealed by EMG," The Williams \& Wilkins Comp., Baltimore, 1985.

[9] C.J. de Luca, "The use of surface electromyography in biomechanics," Journal of Applied Biomechanics, vol. 13(2), pp. 135-163, 1997.

[10] http://www.physionet.org/physiobank/database/emgdb/

[11] C. Berzuini, M.M. Figini, and L. Bernardinelli, "Evaluation of the Effectiveness of EMG Parameters in the Study of Neurogenic Diseases- Statistical Approach Using Clinical and Simulated Data," IEEE Trans. on Biomedical Engineering, vol. 32(1), pp. 15-27, Jan. 1985.

[12] Montana State Hospital Policy and Procedure, "Rehabilation Assessment," RTS-03, pp. 1-3, Dec. 2010.

[13] M. Nikolic, "Detailed Analysis of Clinical Electromyography Signals," Doctoral Dissertaion, University of Cophenhagen, August 2001.

[14] M.B. Bromberg, "Updating motor unit number estimation (MUNE)," Clin. Neurophysiology, vol. 118(1), pp.1-8, Jan. 2007.

[15] X. Li, Y-C Wang, N.L. Suresh, W.Z. Rymer, and P. Zhou, "Motor Unit Number Reductions in Paretic Muscles of Stroke Survivors," IEEE Trans. on Inf. Tech. in Biomedicine, vol. 15(4), pp. 505-512, July 2011. 
International Journal on Bioinformatics \& Biosciences (IJBB) Vol.3, No.3, September 2013

[16] P.G. Ridall ,A.N. Pettitt, R.D. Henderson, and P.A. McCombe, "Motor unit number estimation--a Bayesian approach," Biometrics vol.62(4) pp.1235-50, Dec. 2006.

[17] L.M. Oporto,L.C. Menéndez-de, P.E. Bauzano, M.J. Núñez-Castaín, "Statistical (Poisson) motor unit number estimation," Reviews on Neurology vol. 36(7), pp.601-604, Apr. 2003.

[18] S. Shahid,J. Walker, G.M. Lyons, C.A. Byrne, and A.V.Nene, "Application of higher order statistics techniques to EMG signals to characterize the motor unit action potential," IEEE Trans. on Biomedical Engg., vol.52(7), pp.1195-209, July 2005.

[19] J. Fang, B.T. Shahani, D. Graupe, "Motor unit number estimation by spatial-temporal summation of single motor unit potentials," Muscle Nerve, vol. 20(4), pp.461-8, Apr. 1997.

[20] A.H.Wright, and D.W.Stashuk, "Physiologically Based Simulation of EMG Signals," IEEE Transactions on Biomedical Engineering, vol. 52(2), pp. 171-183, Feb. 2005.

[21] K.S. Türker, "Electromyography: some methodological problems and issues," Phys Ther. vol.73(10) pp.698-710, Oct. 1993.

[22] S. Hargittai, "Savitsky-Golay Least Square Polynomial Filters in ECG Signal Processing," Proc. of Computers in Cardiology Conference, pp. 763-766, Sept. 2005.

[23] Cram J.R., Kasman G.S. and Holtz J., "Introduction to Surface Electromyography," Aspen Publishers Inc., Gaithersburg, Maryland, 1998.

[24] P.K. Artemiadis, K.J. Kyriakopoulos, "Assessment of muscle fatigue using a probabilistic framework for an EMG-based robot control scenario," Proc. of Int. Conf. on BioInf. and BioEng. pp. 1-6, Oct. 2008.

[25] P. Laguna, G. B. Moody, and R. G. Mark, " Power spectral density of unevenly sampled data by leastsquare analysis: performance and application to heart signals,", " IEEE Trans. Biomedical Engineering, vol. 45, no. 6, pp. 698-715, June 1998.

[26] M. Yoshikawa, M. Mikawa, K. Tanaka, "Real-Time Hand Motion Estimation Using EMG Signals with Support Vector Machines," SICE-ICASE, 2006. International Joint Conference, pp. 593-598, Oct. 2006.

[27] L. H. Visser, "Critical illness polyneuropathy and myopathy: clinical features, risk factors and prognosis," European Journal of Neurology, vol. 13, pp. 1203-1212, 2006.

[28] J.N. Hwang, S.S. You, S.R. Lay, and I.C. Jou, "The cascade-correlation learning: a projection pursuit learning perspective," IEEE Transactions on Neural Networks, vol. 7(2), pp. 278-289, Mar. 1996.

[29] J.N.G. Ribeiro, G.C. Vasconcelos, and C.R.O. Queiroz, "A comparative study of the cascadecorrelation architecture in pattern recognition applications," Proc. of IVth Brazilian Symposium on Neural Networks, pp. 31-40, December 1997.

[30] Fahlman, S.E. and C. Lebiere (1990) "The Cascade-Correlation Learning Architecture," Advances in Neural Information Processing Systems, Morgan-Kaufmann, Los Altos CA, 1990.

[31] http://www.noraxon.com/products/instruments/myotrace400.php3

[32] G. Derringer and R. Suich, "Simultaneous Optimization of Several Response Variables," Jour. of Qlty. Tech., vol. 12(4), pp. 214-219, 1980.

[33] T. Fawcett, “An Introduction to ROC Analysis," Pattern Recognition Letters, vol. 27, no. 8, pp. 861874, June 2006.

[34] Christodoulou C.I., Pattichis C.S., 1995, "A New Technique for the Classification and Decomposition of EMG signals", in Proc. IEEE Int. Conf. on Neural Networks, vol. 5, pp. 2303-2308, Nov. 1995.

[35] H.B. Xie, Z. Wang, H. Huang, and C. Qing, "SVM in Computer Aided Clinical EMG," $2^{\text {nd }}$ Int. Conf. on M.L. and Cyb., pp. 1106-1108, 2003.

[36] H.B. Xie, H. Huang, and Z. Wang, “A Hybrid Neurofuzzy System for Neuromuscular Disorders Diagnosis," IEEE Workshop on Biomedical Circuits and Systems, Sec. 2.5, pp. 5-8, 2004. 
International Journal on Bioinformatics \& Biosciences (IJBB) Vol.3, No.3, September 2013

\section{Authors}

Sailesh Conjeti holds a Bachelor of Engineering (Hons.) Degree in Electrical and Electronics Engineering from Birla Institute of Technology and Science, Pilani. He is currently with the School of Medical Science and Technology at Indian Institute of Technology, Kharagpur pursuing his Masters in Medic al Imaging and Informatics. His research interests include Medical Image Computing, Biomedical Signal Processing, Wearable Computing and Rehabilitation Engineering. He has participated in 5 research projects and has 8 publications to his credit.

B. K. Rout completed his B. E. in Mechanical Engineering from University College of Engineering, Burla, Sambalpur (Deemed University) in the year 1990 and completed M. Tech, in Quality, Reliability and Operations Research from Indian Statistical Institute Calcutta, 1992. After graduation he worked with Escort Ancillaries and MESCO Steel Projects for 5 years. He joined BITS Pilani, in December 1998. For the last 12 years he is working as a Faculty member of Mechanical Engineering Group. While serving as a faculty member in the department of Mechanical Engineering, he completed his doctoral research in area of manipulator design under the guidance of Prof. R K Mittal in 2006. So far he has published many research papers in National and International Conferences and in International Journals. His areas of interests are Simulation and Optimization of Dynamic Systems, Design Optimization and Quality Engineering.
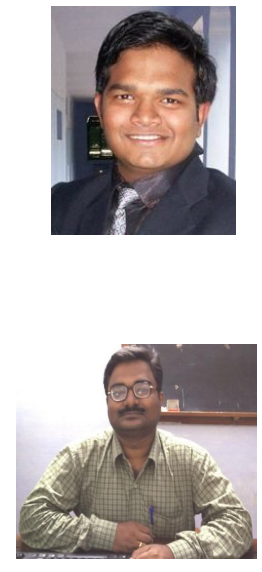